\title{
MORPHOLOGICAL ROAD SEGMENTATION IN URBAN AREAS FROM HIGH RESOLUTION SATELLITE IMAGES
}

\author{
R. Gaetano*, J. Zerubia \\ Ariana Research Team, INRIA \\ Sophia Antipolis, France
}

\author{
G. Scarpa, G. Poggi \\ University Federico II \\ Naples, Italy
}

\begin{abstract}
High resolution satellite images provided by the last generation sensors significantly increased the potential of almost all the image information mining (IIM) applications related to earth observation. This is especially true for the extraction of road information, task of primary interest for many remote sensing applications, which scope is more and more extended to complex urban scenarios thanks to the availability of highly detailed images. This context is particularly challenging due to such factors as the variability of road visual appearence and the occlusions from entities like trees, cars and shadows. On the other hand, the peculiar geometry and morphology of man-made structures, particularly relevant in urban areas, is enhanced in high resolution images, making this kind of information especially useful for road detection.

In this work, we provide a new insight on the use of morphological image analysis for road extraction in complex urban scenarios, and propose a technique for road segmentation that only relies on this domain. The keypoint of the technique is the use of skeletons as powerful descriptors for road objects: the proposed method is based on an ad-hoc skeletonization procedure that enhances the linear structure of road segments, and extracts road objects by first detecting their skeletons and then associating each of them with a region of the image. Experimental results are presented on two different high resolution satellite images of urban areas.
\end{abstract}

Index Terms - Segmentation, classification, skeletonization, pattern recognition, shape analysis.

\section{INTRODUCTION}

With the always increasing availability of high-resolution images of the Earth surface, research on techniques for image information mining in the remote sensing domain is rapidly gaining attention [1]. A particularly affected topic is the one concerning the retrieval of road informations from remotely

\footnotetext{
*This work has been carried out during the tenure of a post-doctoral fellowship (Gaetano's post-doc) funded by the ERCIM consortium, which the authors would like to thank. R. Gaetano is currently covering a post-doctoral position at Telecom ParisTech, France, within the Multimedia Group, dept. of Signal and Image Processing (TSI).
}

sensed data for several different applications, from automatic GIS (Geographic Information System) update [2] to the tracking of changes, urban planning and emergency response applications.

This problem is not of recent interest in literature, since we can find works on the subject that date back to the 1970s [3], and passed through several generations of acquisition systems with radical methodological and application changes [4, 5]. In former works based on mid-resolution sources, the solution has been often limited to the detection of non-urban or main urban roads, like in [5], while in more recent times the attention moved on high resolution imagery, with the development of several automatic and semiautomatic techniques for urban roads detection $[6,7,8,9]$. Different methodologies and approaches have been proposed, from tracking based methods [10], where the detection process relies on the generation of seeds that help following the road structures along the images, techniques based on shape analysis [11], to the methods based on higher order active contours, like in [12, 13], that typically need a higher computational effort. To not forget are also general segmentation techniques that whether properly defined can provide road extraction as a by-product, as is the case of recent texture-based segmentation techniques [14, 15]

Since many of these methods, but the works of Peng's et $a l$. [12,13], above all those aiming at the automatic extraction of road information, has proved their potentials only in simple scenarios, and are likely to fail in complex environments like dense urban areas, in this paper we decided to take back into consideration the use of morphological analysis to cope with this problem, and present here a technique of road segmentation that completely relies on such image processing domain. This choice is justified by the fact that the current resolutions provide data with a considerable richness in fine details, that implicitly enhances the geometric properties of the detected scenes.

\subsection{The problem of urban road segmentation}

Let us discuss this point by taking a look at the images of Fig. 1: in the $4 \mathrm{~m}$ resolution image of Fig. 1(a), roads present a limited width, sometimes not negligible like in the case of main roads, but generally small enough to consider the 


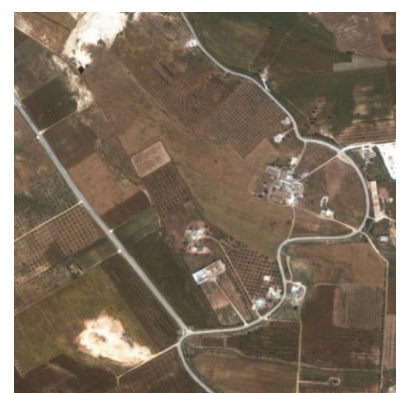

(a)

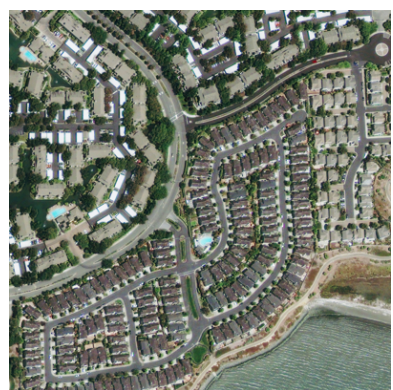

(b)
Fig. 1. Non-urban vs. urban remote sensing data for road extraction: (a) a $4 \mathrm{~m}$ resolution Ikonos image (Copyright (c)Ikonos) of a countryside scene and (b) a Geoeye-1 image (Copyright (C) GeoEye) of an urban area with $0.5 \mathrm{~m}$ resolution. Both images are $900 \times 900$ pixel sized, for scale comparison.

objects of interest as some sort of "thick lines". Moreover, in non-urban contexts roads visibility is often naturally enhanced. In the right image of Fig. 1(b), which exibits a submetric resolution and depicts a urban scenario, road visual appearence is quite different under multiple aspects: amplitude and variation in roads width is significant, as well as for their color, and the presence of occlusions and superpositions from other objects (trees, cars, shadows, etc.) severely compromises the regularity of visible road shapes. Moreover, in urban areas, it is frequent to have the presence close-mesh road networks made of short segments, roundabouts, pedestrian roads or paths of few pixels width, and other typically urban viability structures that could make many of the current state-of-the-art methods unsuited for the case. On one side, then, most of the assumptions (spectral coherence, thinness, quasi-continuity, smoothness, etc.) made in the past are too rigid for urban scenes like the one of Fig. 1(b), while on the other side road objects still show some morphological peculiarity: roads are visually percepted as objects that spread somehow over large parts of the scene, and whose minimum local width at any point is much lower that their total length.

\subsection{Introduction to the proposed method}

In most of the road extraction literature, segmentation often represents a basic step of the process [2], aimed at retrieving the portions of image that could correspond to road surfaces. In cases where these latter are analysed by means of their spectral and textural appearence, this approach is correct if there exists a reliable way to model them, but in environments where such visual appearence changes considerably some problems can arise. On the other hand, some form of segmentation is necessary to create objects to be associated with road segments, for example mining on their shape properties.

Since a complete partition of the image can be too constraining, here we decide to start from the detection of edges

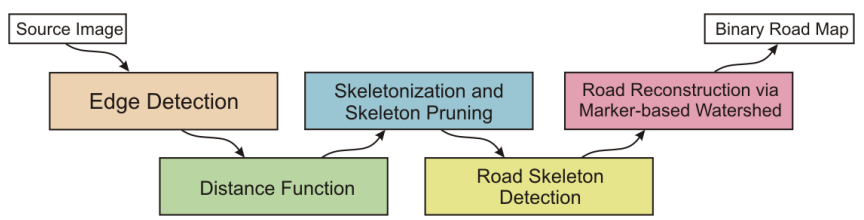

Fig. 2. Flowchart of the proposed algorithm for road segmentation.

and provide a "global" skeletonization of the edge map that will be the basis for the analysis of the morphological content of the image. From this point on, attention is moved to the skeleton image: road sub-skeletons are extracted based on some morphological criteria, mainly based on the idea that the points of a road bodies typically stand close to their skeleton. Once the road skeletons are estimated, complete roads are segmented from the image by means of some morphological reconstruction. It is worth noticing that the obtained segmentation map can be consistently used as the preliminary extraction step in more high-level road detection techniques.

The paper is structured as follows: in section 2 , the proposed method is presented in details, experimental results are then introduced in section 3 , while in section 4 conclusion are given and future work is delineated.

\section{PROPOSED METHOD}

An overall scheme of the proposed algorithm is presented in Fig. 2. The core of the proposed road extraction method is skeletonization. The use of morphological skeletons is a rather natural choice for the analysis of objects as roads: in urban scenes like those addressed in this work, roads are objects that, even if characterized by a non negligible thickness, exibit a mainly linear behaviour through the image. In the ideal case of roads with smooth and parallel contours, their skeleton is represented by a thin line that can without flaw be considered as a linear approximation of the road itself, retaining much of the information of the original object (only width is neglected). Something different happens for other objects in the scene (e.g. building, trees, cars, etc.), whose reliable morphological description would better rely on a contour based representation.

In real cases, object contours, including road edges, present many discontinuities. In general, skeletonization is not robust with respect to such kind of noise, but the effects on the process are less detrimental when applying it to objects like roads: branches due to contour discontinuities are just small appendices connected to the skeleton backbone, while partial occlusions only cause slight deviations on the skeleton line. Such effects can be observed in Fig. 3(c). A simple solution to remove these artifacts will also be presented.

Before getting into the details of the process, an overall insight on how the technique works can be observed in Fig. 3. 


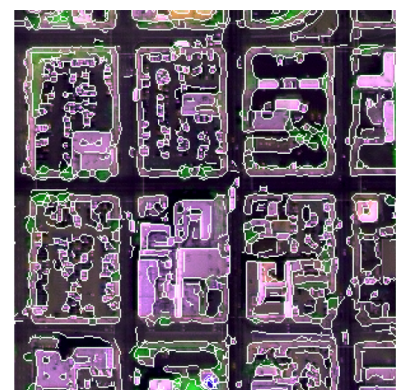

(a)

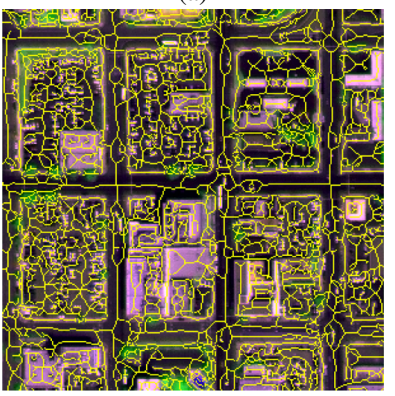

(c)

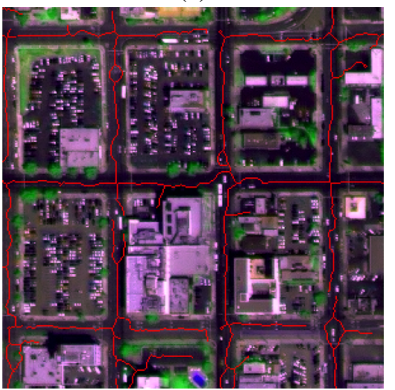

(e)

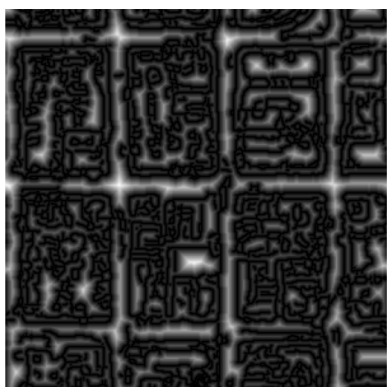

(b)

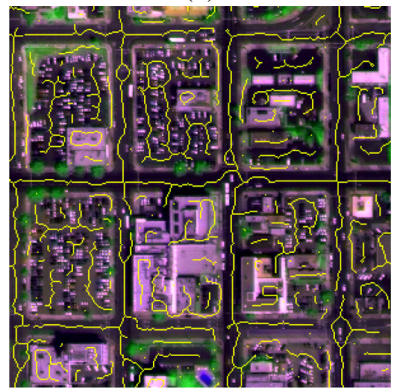

(d)

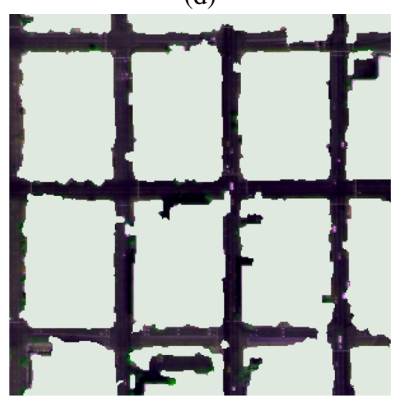

(f)
Fig. 3. An example of road segmentation with the proposed method: (a) a detail of the source image with superimposed contours, (b) result of the distance function computed starting from the contours in (a), and (c) the corresponding skeletonization obtained, (d) result of skeleton pruning, (e) road skeleton selection, (f) final binary road map.

At the first step, edges are extracted from the image as a starting point for the morphological analysis, see Fig. 3(a). Such contours are used to compute a distance function of the edge map, in Fig. 3(b), that is then used to extract the skeleton of the source image depicted in Fig. 3(c). Such skeleton is clearly overloaded, unavoidable effect due to the complexity of scene details and the presence of noise, so a skeleton reduction based on simple morphological criteria is applied resorting to the significant skeleton of Fig. 3(d). The different connected skeleton segments are then indipendently analysed and road skeletons are extracted using shape criteria, further reducing the original skeleton to the one in Fig. 3(e). Based on the available distance function, a watershed-based reconstruction of the image using the different skeleton segments as markers provides the desired road map of Fig. 3(f). No- tice how the chosen morphological diffusion is able to also fill some of the gaps present in the road skeleton, although there are still some other ones to be removed.

In the following, the different step of the segmentation process are illustrated in further detail.

\subsection{Edge Detection}

For the proposed technique, the edge detection phase does not represent a step of high criticity, since it is only used to allow for the analysis of the morphological content of the image. For this step, a basic Canny edge detector [16] is used. This choice is mainly due to the fact that such technique has a reduced computational burden, also for multispectral images since each band is operated independently, and in general a limited number of parameters to set. In addition, we also implemented the simple automatic selection procedure for the low and high threshold described in [16], that takes as high threshold $T_{\text {high }}$ the one that excludes the $70 \%$ of the (Sobel) gradient points after nonmaxima suppression, and consequently sets the low threshold $T_{\text {low }}=0.4 * T_{\text {high }}$. The choice of the smoothing parameter $\sigma$, constant in our case, has proved to have a reduced impact on the final result. Edge maps from the different channels are merged and the result is finally thinned using the hit-and-miss tranform with suitable structuring elements to produce thin edges [17].

\subsection{Distance Function}

The skeletonization technique used for this method is based on the detection of crest lines over the distance function [17], obtained starting from the available edge map. Here we use a simple propagation technique to compute a step-wise Euclidean distance function, in which 8-connected paths are considered but diagonal steps have length equal to $\sqrt{2}$. This reduces the generation of ridge artifacts near smooth curves and provides more precise distance values for further use in the featuring/selection step.

The propagation technique is queue-based. Given $D T$ the desired distance transform, $Q$ a queue of locations, $p, q$ two generic locations, $\mathcal{N}_{4}, \mathcal{N}_{8}$ the 4- and 8-connected neighborhoods, the procedure can be described by the pseudo-code in Fig. 4: The initial distance transform $D T_{0}$ is computed as shown in Fig. 4(a) and (b): locations corresponding to the initial edge pixels are marked as at distance 1 from the contour, following the idea that the real contour is one of the red or green dashed lines of Fig. 4(b). All the locations that touch the ideal contour only with a corner are assigned a value of $\sqrt{2}$ according to the Euclidean metric. This operation is realized by means of a suitable hit-and-miss transform. In Fig. 4(b) the two couples of detected locations, corresponding each to a different "idealization" of the real contour, are marked in red end green accordingly. In Fig. 4(c) the final distance transform of the considered edge sample is shown. The final 


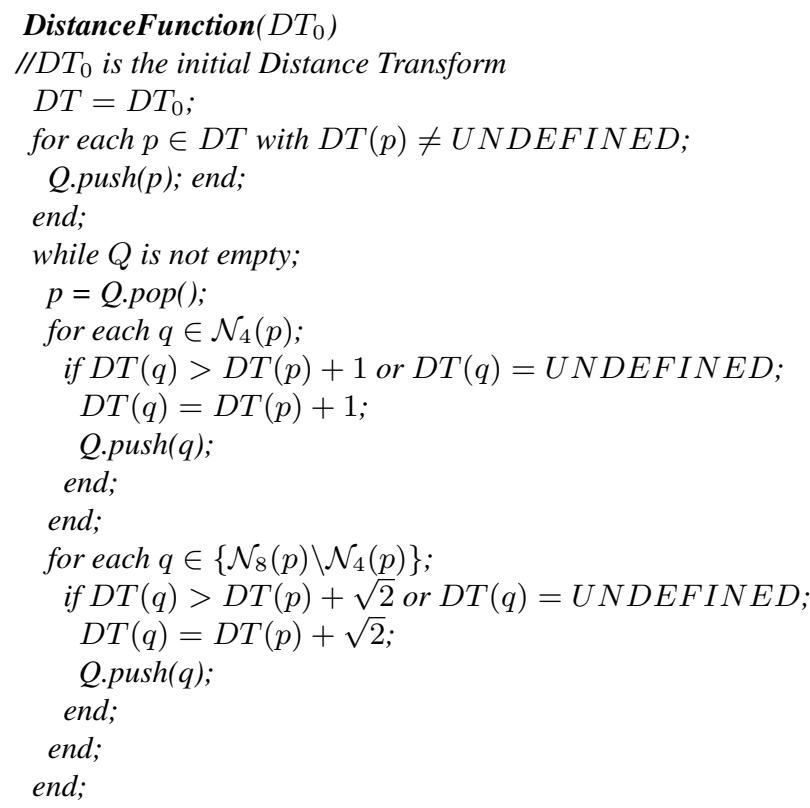

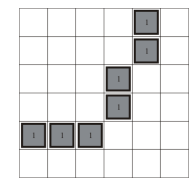

(a)

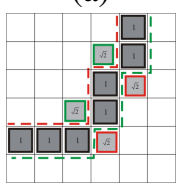

(b)

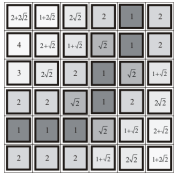

(c)

Fig. 4. Distance function: on the left, the algorithm in pseudo-code. On the right, (a) the original 8-connexity contour detected, (b) placement of the $\sqrt{2}$-valued "curve seeds", (c) result of the distance transform after propagation.

distance transform $D T$ is transformed into an integer-valued matrix by means of a suitable scalar quantization.

\subsection{Skeletonization and Skeleton Pruning}

As already mentioned, the skeletonization procedure here used is based on crest line following over the distance function obtained through the described method. As well known in morphological image analysis, skeletonization can not be considered an exact operation, since any formal definition of skeleton makes sense only in a continuous topology and must be approximated in the discrete case. In this work we follow this rationale: road skeletons are relatively "simple", meaning that their informative potential is enhanced when the result of the skeletonization procedure is reduced to the essential. A simple smooth line is all we need, at least for 1-dimensional representations, to feature a road object. For this reason we decided for an approach that, even if penalizing the precision of the detection, guarantees a fast execution and a simplified result.

More precisely, crest line detection is here based on the following principle: focusing on its local pixel configurations, crest lines of the distance function present points with a characteristic 8-connected neighbourhood, in which it is reasonable to assume that a maximum of two points, in the great majority of the cases, have a higher value. In fact, local maxima (that is, no higher-valued neighbours) are evidently points of a crest line, a single peak in the simplest case; ascending/descending crest lines have in general only one higher neighbour, in the direction of the slope. In constant-altitude crest lines, irregularities can generate 1-pixel saddle points

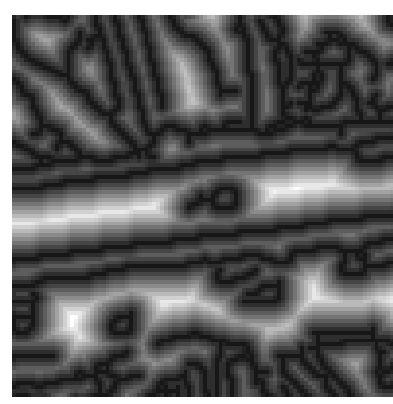

(a)

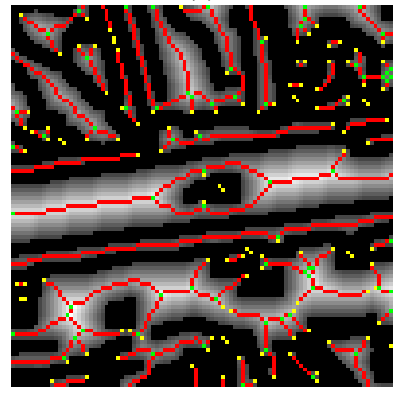

(c)

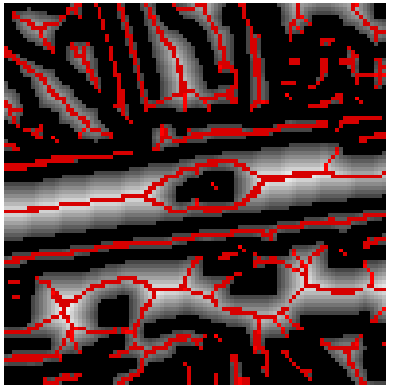

(b)

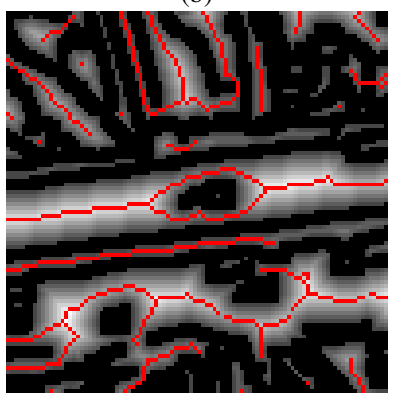

(d)
Fig. 5. Steps of the skeletonization procedure: the original distance transform (a) is first thresholded (the value of the threshold is set to 3 ), then the crest line detection and linking procedure provide the superimposed map in (b) (in bright red the linking points). The thin skeleton in (c), where leaves and nodes are marked respectively in yellow and green, is then pruned to obtain the final result in (d). 
(higher values in two opposite locations of the neighbourhood).

The skeletonization algorithm is based on these main steps: first, the distance transform is thresholded to avoid the proliferation of skeleton points concerning small objects and noisy areas, by setting to zero altitude all the points strictly below a "small" threshold, under which morphological properties of objects are clearly unreliable. Then, all the distance function points having a maximum of two higher neighbours are marked as crest line points. The queue-based skeleton linking technique described in [18] is here applied to complete the crest line detection, that recursively adds to the skeleton the neighboring points in the direction of the steepest ascent. The procedure can be followed in Fig. 5, the result of the preliminary detection and linking being reported in Fig. 5(b). A morphological thinning produces the one pixel wide skeleton of Fig. 5(c).

\subsection{Graph-based Skeleton Pruning}

The last step of the skeletonization procedure is aimed at providing a further simplification of the skeleton in order to clean it from useless information and enhance, where present, objects of the image with a mainly linear structures. In particular, a pruning of the current skeleton is realized by cutting out all its pending branches. In road skeletons, this operation has a very limited effect, since tipycally these objects present a long backbone with small appendices corresponding to contour irregularities or artifacts on the extremities, making this choice useful for our case.

The effect of such pruning can be seen by comparing the images in Fig. 5(c), where leaves and internal nodes of the thin skeleton are marked respectively in yellow and green, and (d), where the pruned skeleton is shown. Linear skeletons keep an almost unaltered aspect, while the pruning mostly affects small skeletons with a mainly starry structure. The pruning step is here realized by first vectorizing image skeletons into abstract undirected graph structures, that associates each skeleton branch with an edge of the graph. Seeking for nodes with connection degree equal to one and removing the only corresponding edge, together with the corresponding branch of the skeleton, is then a trivial operation.

This vectorization procedure is clearly not crucial for the considered scope, since a more lightweight queue-based procedure could have provided a similar result. Anyway, we decided to present this algorithm as a further contribution of this paper, since it represent an effective tool to provide an abstract representation of image content and opens to many higher level possibilities in terms of image analysis and mining. A pseudocode version of the vectorization algorithm is reported in Fig. 6. Starting from the available binary map ( $\left.s k_{\_} m a p\right)$, once the endpoints (leaves) and the intersection points (nodes) of the skeleton are detected, issue of well known morphological operators [17], they are picked one by one and the corre- sponding connected skeleton is traversed, by following each branch (a set of contiguous points between two graph nodes) and recording it as a link on the graph. At each WalkSkeleton iteration a set of multiple leaves and nodes are traversed and excluded by the next picking round. An example of skeleton vectorization is depicted in Fig. 7.

\subsection{Roads Detection and Map Reconstruction}

To extract areas of the image that can be associated with roads, the exclusive use of morphological indicators is here taken into consideration. Road fragments are here analysed starting from their skeleton: each connected skeleton segment of the image, that is, a set of adjacent skeleton pixels, is here considered as a whole and featured indipendently.

Thanks to the aforementioned suitability of the skeleton in tracking roads, a quite simple featuring strategy can be used to determine whether a skeleton segment is belonging to a road or not. Based on the straightforward consideration that a road segment has a considerable length w.r.t. its width, a figure (the road skeleton score, RSS) is associated to each of them that relies on the ratio between the average distance function and the total length of the segment. For the $i$-th skeleton segment $\mathcal{S}_{i}$ containing $\left|\mathcal{S}_{i}\right|$ pixels, being $\mathcal{D}$ the distance function and $\mathcal{L}_{i}$ is the skeleton length:

$$
R S S_{i}=\frac{\overline{\mathcal{D}}_{i}}{\mathcal{L}_{i}} \quad \text { where } \quad \overline{\mathcal{D}}_{i}=\frac{\sum_{p \in \mathcal{S}_{i}} \mathcal{D}(p)}{\left|\mathcal{S}_{i}\right|}
$$

The skeleton length $\mathcal{L}_{i}$ is obtained as a byproduct of the vectorization procedure in Fig. 6, by cumulating step length of size 1 for horizontal and vertical movements and size $\sqrt{2}$ for diagonal movements. $\overline{\mathcal{D}}_{i}$ is the average distance function over the skeleton points.

Selection is made by means of a double thresholding, by keeping as road skeletons all the segments $i$ that verify the two conditions $R S S_{i}<R S S_{t h}$ (2) and $\overline{\mathcal{D}}_{i}<\overline{\mathcal{D}}_{t h}$ (3). The condition (2) identify objects in the image with a low ratio between the average width and the length, while the condition (3) helps to prevent the selection of non-linear objects that present many holes, for example large background regions. In this case, infact, skeletons present loops that contribute considerably to the total skeleton length, but their average distance function remains typically higher with respect to road segments.

The map reconstruction step is realized by applying the marker based watershed transform [17] on the distance function obtained by inverting the distance function, using the pruned skeleton as marker image. This virtually reconstructs a segmentation map, with each connected component containing exactly one connected skeleton segment. The fragment selected as road segments are those associated to the selected skeletons. 


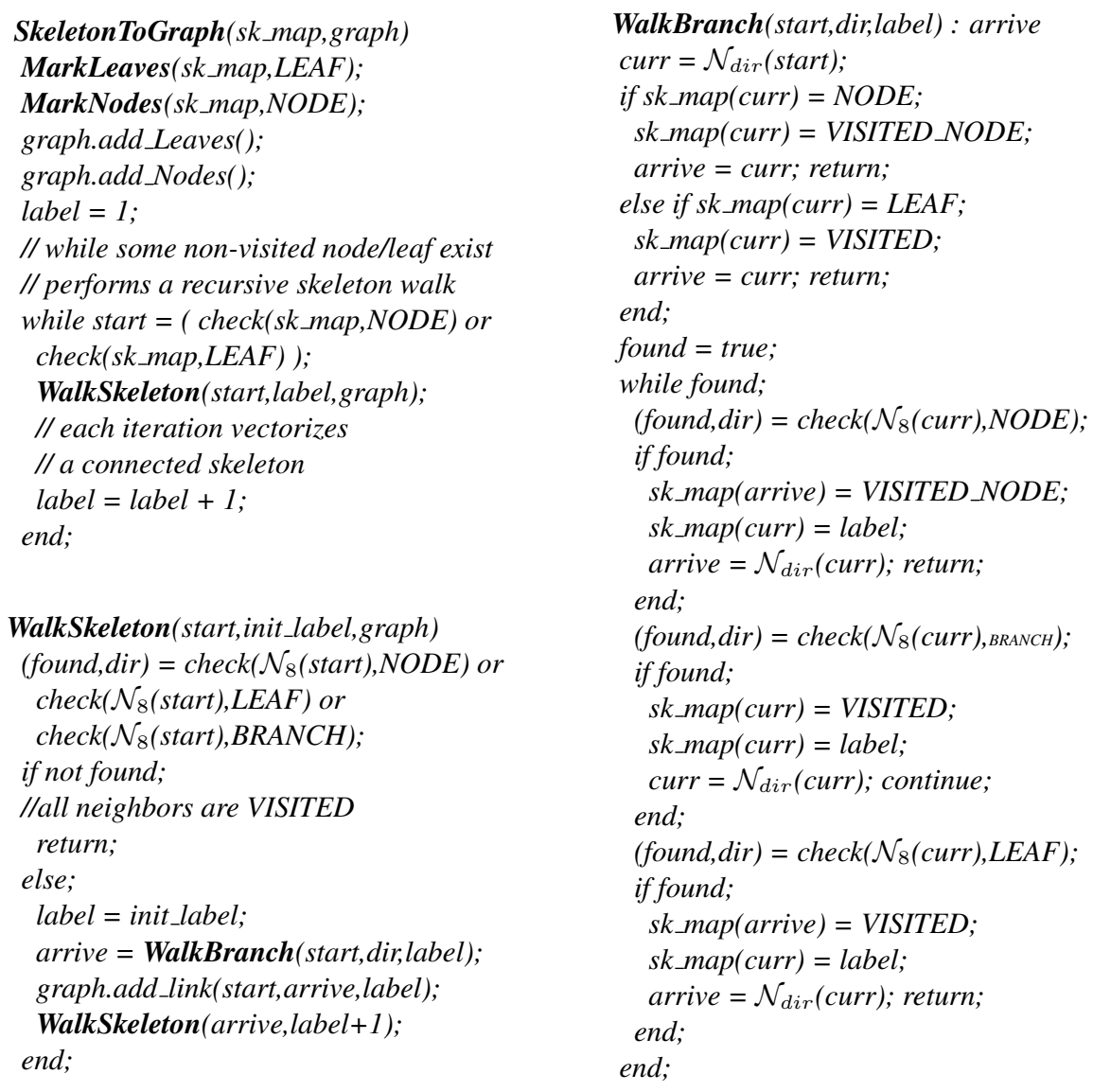

Fig. 6. Pseudo-code for the skeleton vectorization procedure. In the skeleton map sk_map, the points are initially labeled as $L E A F, N O D E S$ or BRANCH according to their position. $\mathcal{N}_{\text {dir }}(p)$ indicates the 8-connected neighbour of $p$ in the direction dir .

\section{EXPERIMENTAL RESULTS}

The described technique has been tested on two different high resolution satellite images of complex urban scenarios. The first is a $2004 \times 2004$ image of San Diego (USA) from the Ikonos satellite, 4-band (near infrared, red, green and blue) pansharpened with $1 \mathrm{~m}$ spatial resolution, reported in false colors in Fig. 8(a). The result of the morphological analysis described in the previous sections is shown in Fig. 8(b), where the values of the thresholds are set to $R S S_{t h}=0.04$ and $\overline{\mathcal{D}}_{t h}=12$. These values are justified by intuitive considerations: for the first one, the condition imposed is that the ratio between the road segment length and its half-width is 25 , while the second one excludes objects with a half-width lower than 12 pixels (assuming a maximum road width of $1 m \times 12 \times 2=24 m$ for this image).

The result is promising, even if some very thin roads have been missed probably because the corresponding skeleton is highly fragmented. Notice that most of the classification errors (false positives) concerns thin vegetation areas, often adjacent to the streets, that present a pronounced linear shape. In Fig. 8(c) the fragments of the image, obtained by the described watershed-based reconstruction, with a positive

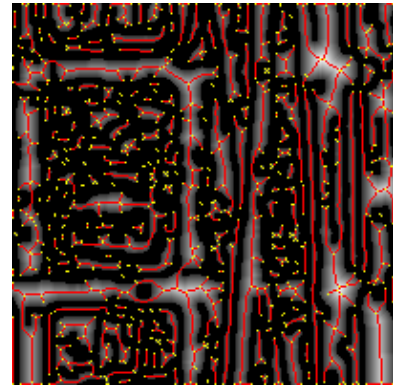

(a)

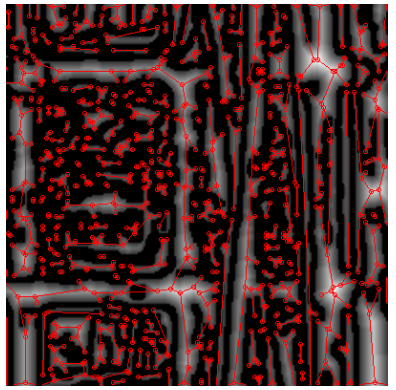

(b)
Fig. 7. Skeleton vectorization: (a) the skeleton map with highlighted endpoints and intersections, (b) the corresponding graph obtained using the procedure in Fig. 6.

NDVI vegetation index are removed from the result. This shows the potential of the proposed technique in combination with other approaches that make use of features of different nature.

The second row of Fig. 8 shows the result of the proposed segmentation technique over a $0.41 \mathrm{~m}$ resolution RGB image of San Francisco, that shows a very complex urban sce- 


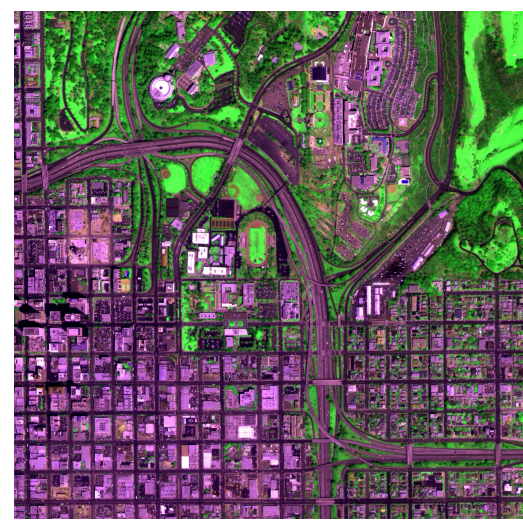

(a)

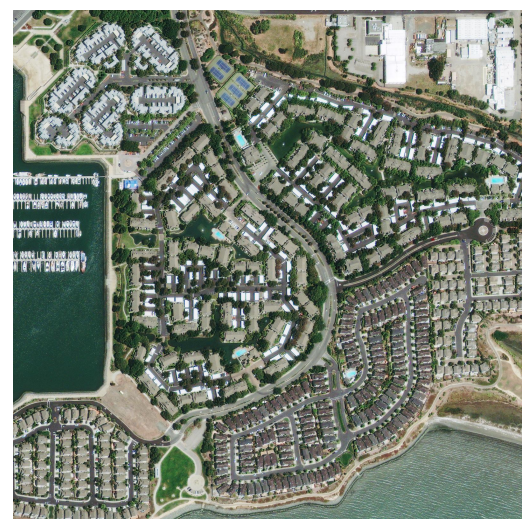

(d)

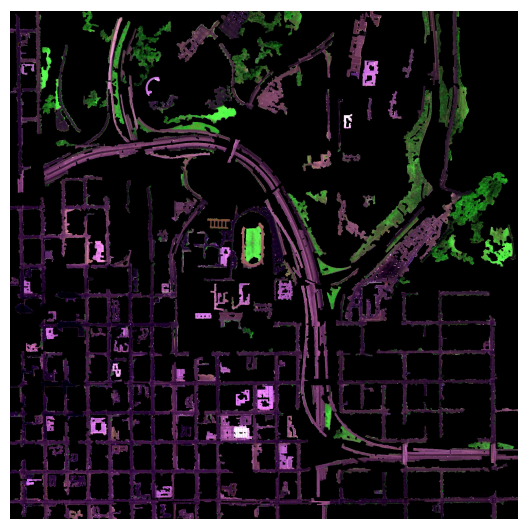

(b)

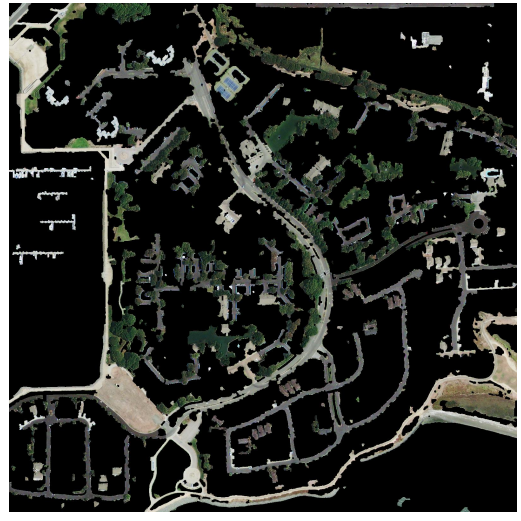

(e)

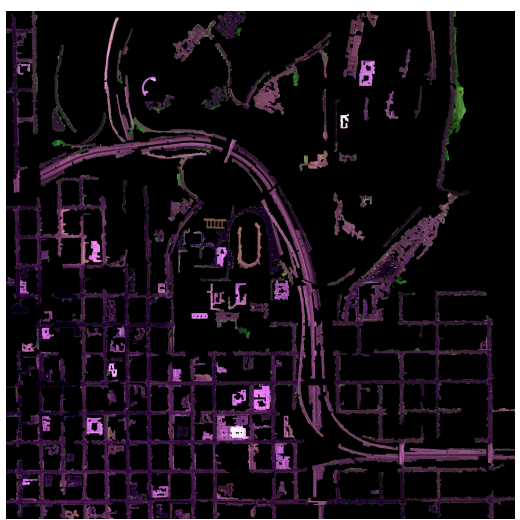

(c)

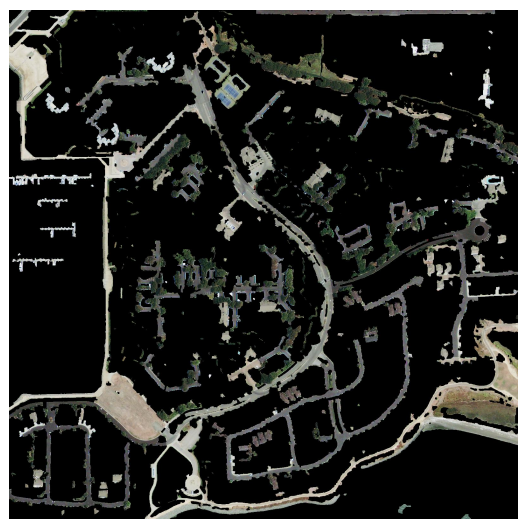

(f)

Fig. 8. Experimental results. (a) A $1 \mathrm{~m}$ resolution 4-band pansharpened image of San Diego, CA, USA, from the Ikonos satellite (Copyright (CIkonos), (b) road segmentation with the proposed method, (c) enhanced result via vegetation removal. (d) A $0.41 \mathrm{~m}$ resolution RGB image of San Francisco, CA, USA, from the GeoEye-1 satellite (Copyright (C)GeoEye), (e) road segmentation result and (f) enhancement. For both experiments, the values of the thresholds are $R S S_{t h}=0.04$ and $\overline{\mathcal{D}}_{t h}=12$.

nario with roads of multiple widths, colors and structures (e.g. roundabouts). Results (see Fig. 8(b)) are again quite promising, and the high resolution allows here also for the extraction of very thin pedestrian roads and paths, whose skeleton is better defined with respect to the previous image. Again, many errors come from adjacent vegetation: since the near infrared channel is not available for this image, a simpler green index is here used, defined as $G I=G /(R+G+B)$, and all the fragments whose average $G I$ is above a threshold of 0.4 are removed. The enhanced result is shown in Fig. 8(f).

\section{CONCLUSIONS}

In this paper, the use of morphological image analysis for the extraction of road information from high resolution remote sensing images has been inspected, and a technique of road segmentation presented that only relies on morphological properties. The technique is based on the subsequent application of several morphological operators: edges are extracted from the source image and a distance function is com- puted. A suitable skeletonization procedure is then applied that retrieves image skeleton and simplify it by neglecting small objects and removing branches to enhance the linear nature of road skeletons. Single connected skeletons are then featured using a suitable metric and classified by a double thresholding. Image reconstruction based on the watershed transform, with skeletons used as markers, finally provides the road segmentation map.

Experimental results proves the effectiveness of the morphological properties in conveying road information from even complex urban scenarios, where the unpredictable nature of road visual aspect makes the use of a rigid modeling strategy sometimes less effective. The promising nature of these preliminary results will lead towards a further inspection in the potential of this method, mainly through the graph-based analysis of the vectorized skeleton, and the modification of the skeletonization procedure to address the detection of other entities of interest. 


\section{REFERENCES}

[1] G. Masi, R. Gaetano, G. Scarpa, and G. Poggi, "Dynamic segmentation for image information mining," in Proceedings of IEEE International Geoscience and Remote Sensing Symposium, Honolulu, HI (USA), July 2009, pp. $1992-1995$.

[2] J.B. Mena, "State of the art on automatic road extraction for GIS update: a novel classification," Pattern Recognition Letters, vol. 24, pp. 3037-3058, 2003.

[3] R. Bajcsy and M. Tavakoli, "Computer recognition of roads from satellite pictures," IEEE Transactions on Systems, Man and Cybernetics, vol. 6, pp. 623-637, 1976.

[4] D. Geman and B. Jedynak, "An active testing model for tracking roads in satellite images," IEEE Transactions on Pattern Analysis and Machine Intelligence, vol. 18, no. 1, pp. 1-14, January 1996.

[5] N. Merlet and J. Zerubia, "New prospects in line detection by dynamic programming," IEEE Transactions on Pattern Analysis and Machine Intelligence, vol. 18, no. 4, pp. 426-431, April 1996.

[6] C. Lacoste, X. Descombes, and J. Zerubia, "Road network extraction in remote sensing by a Markov object process," in Proceedings of IEEE International Conference on Image Processing, 2003.

[7] C. Lacoste, X. Descombes, and J. Zerubia, "Point processes for unsupervised line network extraction in remote sensing," IEEE Transactions on Pattern Analysis and Machine Intelligence, vol. 27, no. 10, pp. 15681579, October 2005.

[8] V. Pandit, S. Gupta, and K.S. Rayan, "Automatic road network extraction using high resolution multi-temporal satellite images," in Proceedings of IEEE International Geoscience and Remote Sensing Symposium, 2009.

[9] Y. Li, L. Xu, and H. Piao, "Semi-automatic road extraction from high resolution remote sensing image: review and prospects," in Ninth International Conference on Hybrid Intelligent Systems, 2009.

[10] X. Lin, J. Zhang, Z. Liu, and J. Shen, "Semi-automatic road tracking by template matching and distance transform," in Proceedings of Urban Remote Sensing Joint Event, 2009.

[11] P. Gamba, F. Dell'Acqua, and G. Lisini, “Improving urban road extraction in high-resolution images exploiting directional filtering, perceptual grouping, and simple topological concepts," IEEE Geoscience and Remote Sensing Letters, vol. 3, pp. 387-391, 2006.
[12] T. Peng, I.H. Jermyn, V. Prinet, and J. Zerubia, "Incorporating generic and specific prior knowledge in a multiscale phase field model for road extraction from vhr images," IEEE Journal of Selected Topics in Applied Earth Observations and Remote Sensing, vol. 1, no. 2, pp. 139-146, June 2008.

[13] T. Peng, I. Jermyn, V. Prinet, and J. Zerubia, "Extended phase field high order active contour models for networks," International Journal of Computer Vision, vol. 88, no. 1, pp. 111-128, May 2010.

[14] R. Gaetano, G. Scarpa, and G. Poggi, "Hierarchical texture based segmentation of multiresolution remote sensing images," IEEE Transactions on Geoscience and Remote Sensing, vol. 47, no. 7, pp. 2129-2141, July 2009.

[15] G. Scarpa, R. Gaetano, M. Haindl, and J. Zerubia, "Hierarchical multiple Markov chain model for unsupervised texture segmentation," IEEE Transactions on Image Processing, vol. 18, no. 8, pp. 1830-1843, August 2009.

[16] J.F. Canny, "A computational approach to edge detection," IEEE Transactions on Pattern Analysis and Machine Intelligence, vol. 8, pp. 679-698, 1986.

[17] P. Soille, Morphological Image Analysis, Principle and Applications, 2nd ed., Springer-Verlag, 2002.

[18] Y. Ge and J.M. Fitzpatrick, "On the generation of skeletons from discrete Euclidean distance maps," IEEE Transactions on Pattern Analysis and Machine Intelligence, vol. 18, pp. 1055-1066, 1996. 